\title{
Flexible high capacitance gate insulators for organic field effect transistors
}

\author{
L A Majewski ${ }^{1}$, R Schroeder and M Grell \\ Department of Physics and Astronomy, University of Sheffield Hicks Building, \\ Hounsfield Rd, Sheffield S3 7RH, UK \\ E-mail: php01lam@sheffield.ac.uk
}

Received 2 September 2003

Published 10 December 2003

Online at stacks.iop.org/JPhysD/37/21 (DOI: 10.1088/0022-3727/37/1/005)

\begin{abstract}
We have manufactured flexible field effect transistors with both polymeric and low molecular weight organic semiconductors onto a very thin $(6.5 \mathrm{~nm})$ gate insulator with capacitance in excess of $600 \mathrm{nF} \mathrm{cm}^{-2}$. Gate insulators were prepared by anodization of a sputtered aluminium film on a Mylar plastic sheet. Anodization protocols in very dilute acid and in pure water, were explored and results compared.
\end{abstract}

\section{Introduction}

Transistors using organic semiconductors (organic field effect transistors, OFETs) have been the focus of intense research interest. It is envisaged that the processing advantages of organic semiconductors will lead to applications that are either very cheap, or complement organic display technology. Outstanding examples include inkjet- printed logic circuits [1], and organic active matrix drivers for displays [2].

Initially, research and development has mainly focused on the organic semiconductor itself, but recently, contacts [3] and gate insulators for OFETs [4-8] have received increasing attention. An interesting approach to gate insulators are anodically prepared metal oxide films on metal as gate electrode/gate insulator. Tate et al [5] and Iino et al [6] have reported on organic transistors using anodized tantalum $\left(\mathrm{Ta}_{2} \mathrm{O}_{5}\right)$, and we have demonstrated OFETs with anodized aluminium $\left(\mathrm{Al}_{2} \mathrm{O}_{3}\right)$ gate insulators [7]. $\mathrm{Al}_{2} \mathrm{O}_{3}$ has a lower dielectric constant $(\varepsilon)$ than $\mathrm{Ta}_{2} \mathrm{O}_{5}$; however, it has a higher breakdown field and has properties consistent with the demands on organic electronics such as low cost and compatibility with plastic substrates. Anodized Al has been used previously in amorphous silicon FETs [9].

Here we report on OFETs built onto commercial Mylar films coated with a very thin, sputtered Al layer. We have optimized the anodization procedure to manufacture flexible OFETs with gate insulators of $\approx 6.5 \mathrm{~nm}$ thickness, implying very high gate capacitance.

1 Author to whom any correspondence should be addressed.

\section{Experimental}

Our starting point was a commercially available Mylar film coated with a thin, smooth sputtered Al layer. The Al surface had a mirror-like appearance; scanning with a Dektak surface profilometer showed Al thickness of $\approx 60 \mathrm{~nm}$ with surface roughness of $\pm(7-10) \mathrm{nm}$. To produce a gate insulator onto $\mathrm{Al}$, coated Mylar film was cut into stripes of $\approx 4 \mathrm{~cm} \times 2 \mathrm{~cm}$, a wire was attached by conductive glue, and films were immersed into an anodization bath. Two anodization procedures were employed: first, an electrolyte of $1 \mathrm{mmoll}^{-1}$ citric acid in high purity de-ionized water $(18 \mathrm{M} \Omega \mathrm{cm})$ was used, which is less concentrated acid than what was used in previous work [5-7], as we tried to minimize the inclusion of acid counterions into the $\mathrm{Al}_{2} \mathrm{O}_{3}$ film [10]. Anodization was at a fixed current density $j=0.3 \mathrm{~mA} \mathrm{~cm}^{-2}$ maintained by ramping the anodization voltage up to $5 \mathrm{~V}$. Anodization was then stopped and films were washed in de-ionized water. With an anodization ratio $c_{\mathrm{Al}}=1.3 \mathrm{~nm} \mathrm{~V}^{-1}$, this corresponds to an insulator thickness of $6.5 \mathrm{~nm}$. We refer to these films as 'citric acid' insulators. Second, Al-coated Mylar was anodized in a bath containing $18 \mathrm{M} \Omega \mathrm{cm}$ water only, relying on the autoprotolysis of water. This procedure has been introduced by Lin and Hwu [11] for ultrathin silicon nitride films. Films were placed very close $(2 \mathrm{~mm})$ to the cathode to reduce resistance. A constant voltage of $100 \mathrm{~V}$ was applied to drive a (non-constant) anodization current through the growing insulator barrier, and the high resistance bath. In this anodization mode, the thickness of the resulting film is controlled by the duration of anodization (here $15 \mathrm{~s}$ ). We refer to these films as 'water' insulators. We note that 'water' 
anodization becomes difficult for thicker insulator films, as anodization times increase considerably and the prolonged exposure to high anodization voltage may lead to delamination of Al films, even when thicker Al films evaporated to glass are used.

For capacitance measurements with a conventional capacitance meter, circular gold electrodes were evaporated. For 'citric acid' insulators, we found $C_{\mathrm{i}}=620 \mathrm{nF} \mathrm{cm}^{-2}$, and for 'water' insulators, $C_{\mathrm{i}}=(650-700) \mathrm{nF} \mathrm{cm}^{-2}$. This shows that 'water' insulators are of very similar thickness to 'citric acid' films for the anodization protocol described.

We prepared OFETs on 'citric acid' and 'water' insulator films with both polymeric and low molecular weight hole-transporting organic semiconductors. We used regioregular poly(3hexyl thiophene) (rrP3HT) and pentacene, both purchased from Aldrich. $\mathrm{rrP} 3 \mathrm{HT}$ was de-doped with hydrazine before use and was spun from $10 \mathrm{~g}^{-1}$ chloroform solution at $1000 \mathrm{rpm}$. Pentacene was sublimated, as received, under vacuum $\left(<10^{-6}\right.$ Torr) at a rate of $0.4 \mathrm{~nm} \mathrm{~s}^{-1}$ to a thickness between 40 and $110 \mathrm{~nm}$, as measured by a surface profilometer. Transistors were completed by shadow mask evaporation of gold top contacts of $2 \mathrm{~mm} \times 2 \mathrm{~mm}$ separated by a $25-40 \mu \mathrm{m}$ channel. Figure $1(b)$ illustrates the flexibility of the resulting OFETs. For characterization, the source and drain were contacted with Karl Süss probeheads; the contact wire for anodization was used as the gate contact. rrPHT transistors were kept under dynamic rotary pump vacuum for at least $12 \mathrm{~h}$ prior to measurement to reverse the well-known effect of oxygen doping [12]. Output characteristics were measured under nitrogen atmosphere with two Keithley 2400 sourcemeasure units. Gate and drain voltages $\left(V_{\mathrm{G}}\right.$ and $\left.V_{\mathrm{D}}\right)$ were limited to -(3-4) $\mathrm{V}$ to avoid dielectric breakdown of the gate insulator. The maximum breakdown voltage of an anodized film equals the anodization voltage [7], here $5 \mathrm{~V}$.

\section{Results}

Figures 2(a)-(e) show the output characteristics of flexible OFETs using different anodization protocols and semiconductors. To determine the carrier mobility and the threshold voltage, $V_{\mathrm{G}}$ and $V_{\mathrm{D}}$ were ramped up simultaneously and the square root of the drain current $\left(I_{\mathrm{D}}\right)$ was plotted against $V_{\mathrm{G}}=V_{\mathrm{D}}$. Note that at $V_{\mathrm{G}}=V_{\mathrm{D}}$, there is no drain-to-gate leakage current; therefore, mobility determined in this way is not compromised by gate leakage. The measured hole mobility, $\mu_{\text {meas }}$, and threshold voltage, $V_{\mathrm{T}}$, were obtained from the slope and intercept of the resulting straight lines. Table 1 summarizes the results.

\section{Discussion}

Figures 2(a)-(e) show the familiar form of FET output characteristics. For $V_{\mathrm{G}}>V_{\mathrm{T}}, I_{\mathrm{D}}$ initially rises linearly with $V_{\mathrm{D}}$ but saturates as $V_{\mathrm{D}}$ exceeds $V_{\mathrm{G}}$. Transistor action is observed with low operational voltage of less than $-3 \mathrm{~V}$ due to the use of very thin gate insulators with unusually high gate capacitance. For rrPHT transistors, there is a small $I_{\mathrm{D}}$ component rising linearly with $V_{\mathrm{D}}$ even in the saturation regime, or for $V_{\mathrm{G}}$ below $V_{\mathrm{T}}$. This results from residual $\mathrm{rPHT}$ doping, despite hydrazine (a)
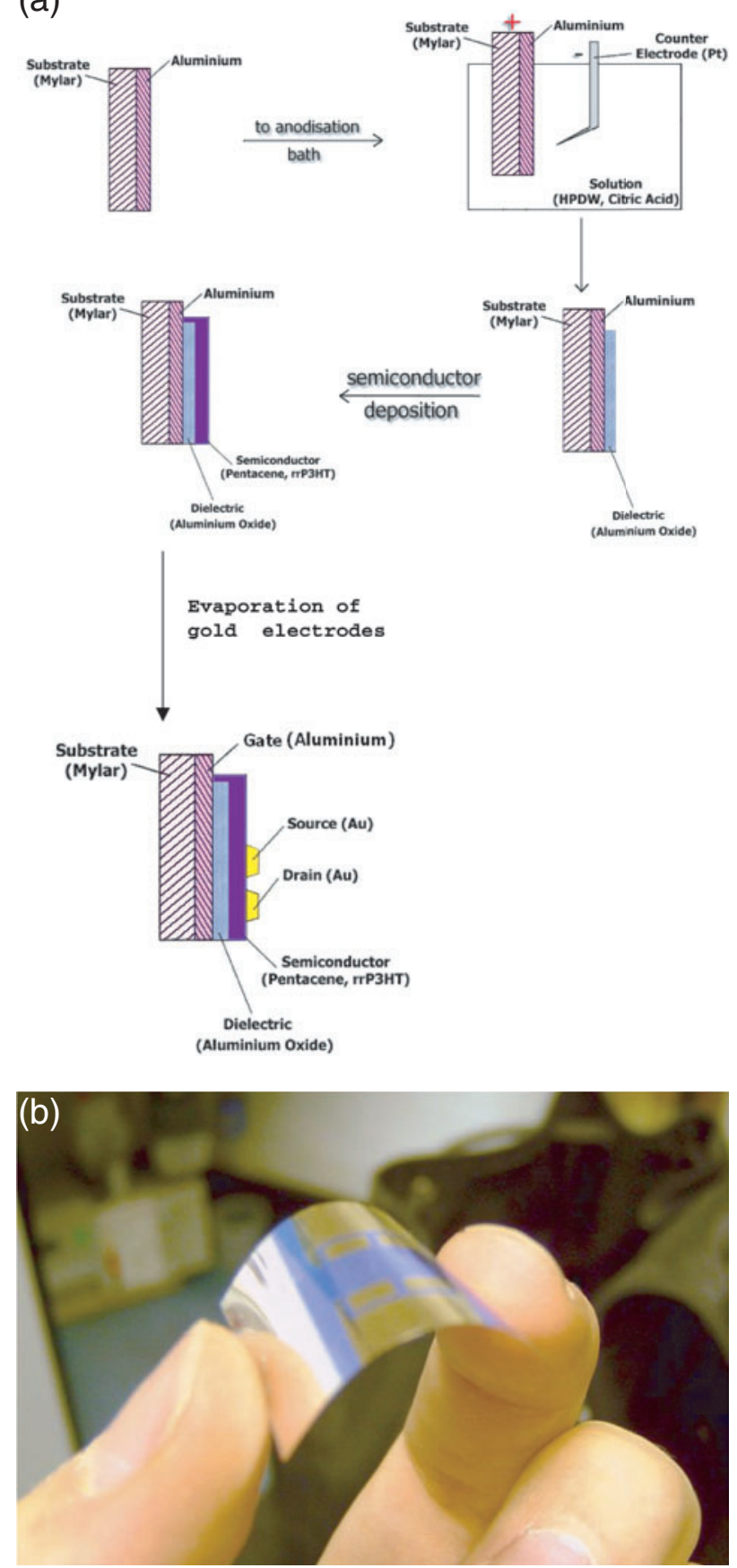

Figure 1. (a) Fabrication steps for flexible organic transistors based on metalized Mylar film; $(b)$ real appearance of tested devices.

de-doping and evacuation, probably due to processing under oxygen [12].

For both rrPHT devices, the threshold voltage is considerably lower than for pentacene transistors. At the gold/pentacene junction, there is an injection barrier of $0.85 \mathrm{eV}$ [13], while the gold/rrPHT junction can be considered Ohmic. We have shown that the injection barrier at the gold/pentacene junction increases $V_{\mathrm{T}}[14]$.

Bao et al [15] reported $\mu=9.2 \times 10^{-3} \mathrm{~cm}^{2} \mathrm{~V}^{-1} \mathrm{~s}^{-1}$ for rrPHT transistors spincast from chloroform onto $\mathrm{SiO}_{2}$ gate insulators and contacted with gold electrodes. This is 


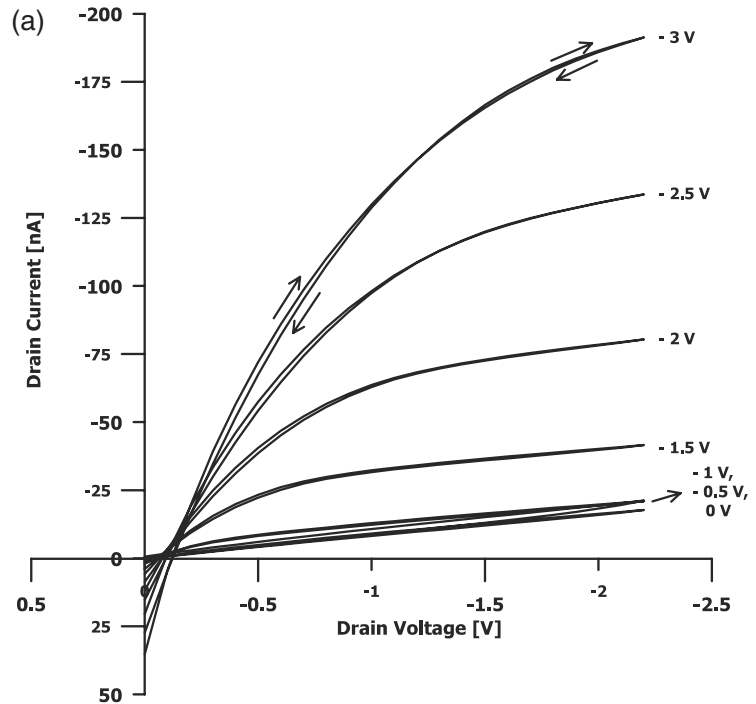

(c)

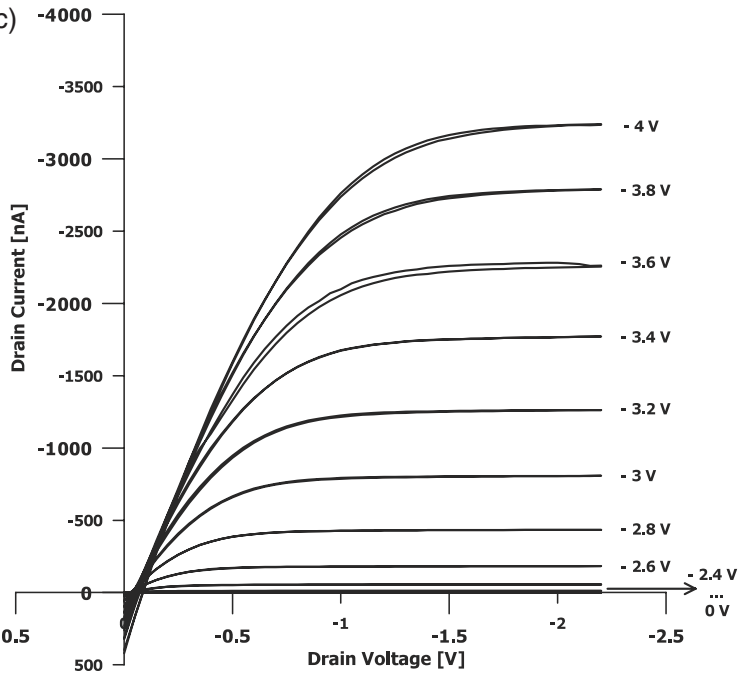

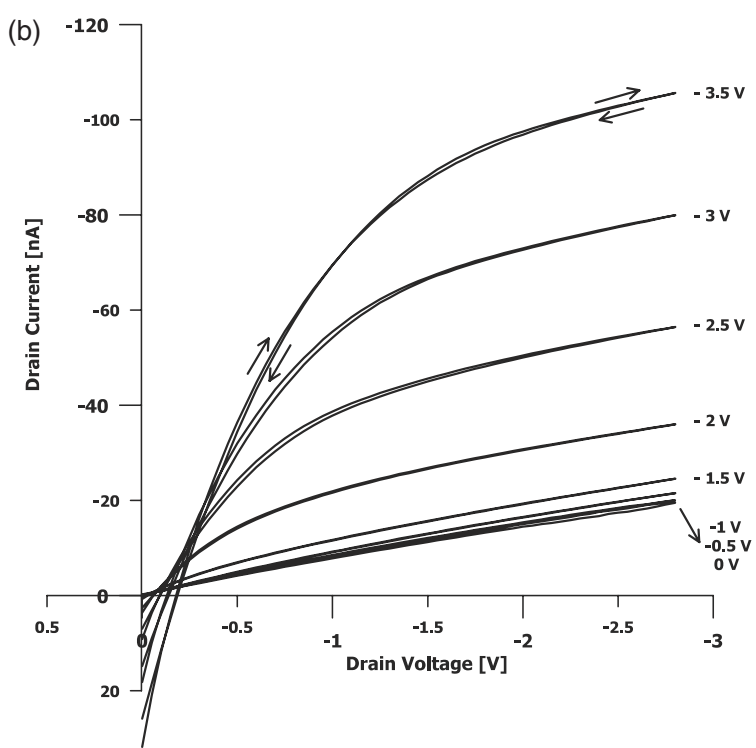

(d)

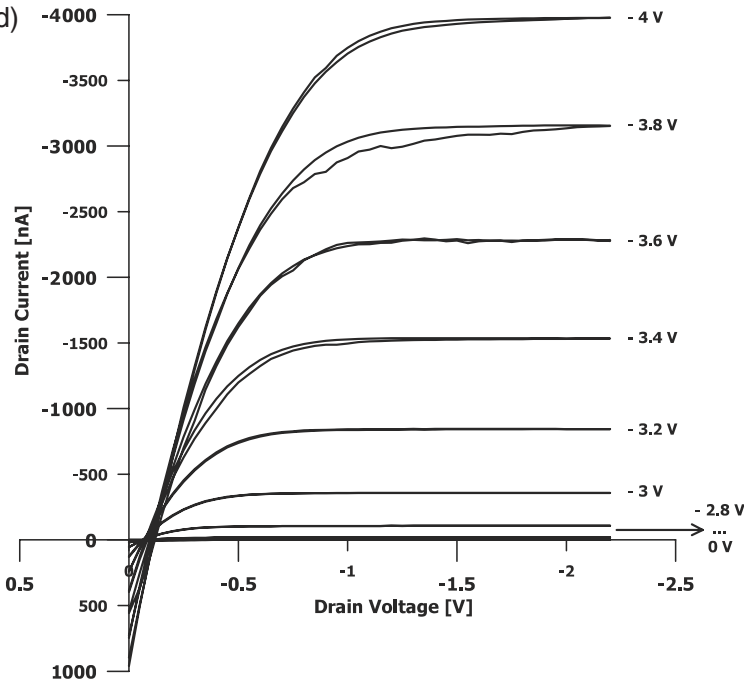

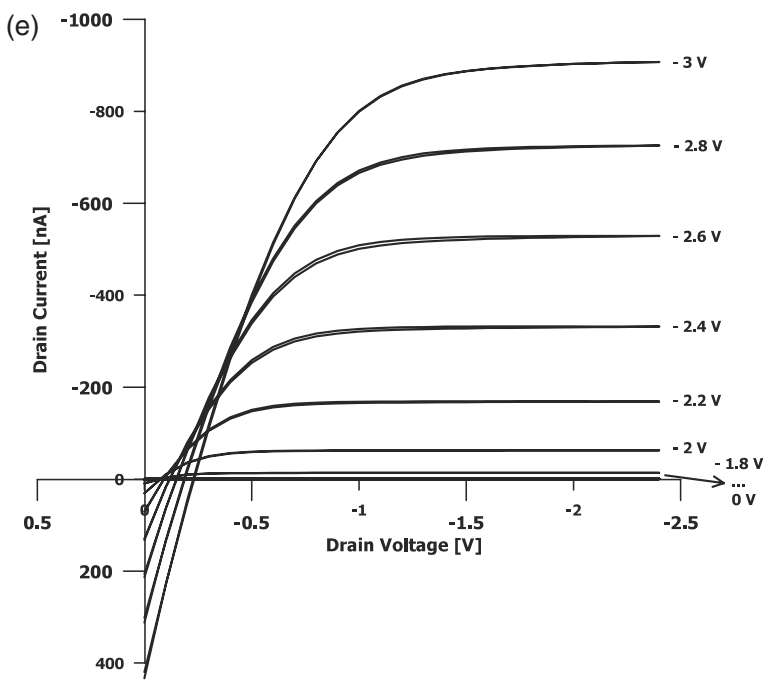

Figure 2. Output characteristics for organic transistors with different semiconductors on anodized Al. (a) rrPHT on water anodized Al—channel length $L=25 \mu \mathrm{m} ;(b)$ rrPHT on citric acid anodized Al—channel length $L=40 \mu \mathrm{m} ;(c) 100 \mathrm{~nm}$ pentacene on citric acid anodized $\mathrm{Al} ;(d) 40 \mathrm{~nm}$ pentacene on citric acid anodized $\mathrm{Al} ;(e) 110 \mathrm{~nm}$ pentacene on water anodized Al. All pentacene devices had channel length $L=25 \mu \mathrm{m}$. 
Table 1. Mobilities $\mu$ and threshold voltages $V_{\mathrm{T}}$ for flexible organic transistors on Mylar substrates/Al gate electrode/anodized $\mathrm{Al}_{2} \mathrm{O}_{3}$ gate insulator using different anodization protocols, and organic semiconductors.

\begin{tabular}{llll}
\hline Semiconductor (figure) & Insulator & $\begin{array}{l}\mu_{\text {meas }} \\
\left(\mathrm{cm}^{2} \mathrm{~V}^{-1} \mathrm{~s}^{-1}\right)\end{array}$ & $\begin{array}{l}V_{\mathrm{T}} \\
(\mathrm{V})\end{array}$ \\
\hline rrPHT $(2(a))$ & Water & $1.39 \times 10^{-3}$ & 0.7 \\
rrPHT $(2(b))$ & Citric acid & $1.14 \times 10^{-3}$ & 0.7 \\
$100 \mathrm{~nm}$ pentacene $(2(c))$ & Citric acid & 0.058 & 2.4 \\
$40 \mathrm{~nm}$ pentacene $(2(d))$ & Citric acid & 0.10 & 2.3 \\
$110 \mathrm{~nm}$ pentacene $(2(e))$ & Water & 0.028 & 1.8 \\
\hline
\end{tabular}

higher than the rrPHT mobility found here. Veres et al [8] have shown that OFET mobility decreases with increasing insulator polarity due to increased energetic disorder at the insulator/semiconductor interface. It is thus not surprising that OFET mobility is lower on $\mathrm{Al}_{2} \mathrm{O}_{3}(\varepsilon=9-11)$ than on $\mathrm{SiO}_{2}(\varepsilon=3.3)$.

The measured mobilities in our pentacene transistors on $\mathrm{Al}_{2} \mathrm{O}_{3}$ are considerably lower than those found for pentacene on anodic $\mathrm{Ta}_{2} \mathrm{O}_{5}\left(\mu=0.24-0.36 \mathrm{~cm}^{2} \mathrm{~V}^{-1} \mathrm{~s}^{-1}\right.$, Iino et al [6]), or pentacene on polymeric gate insulators $\left(\mu=3 \mathrm{~cm}^{2} \mathrm{~V}^{-1} \mathrm{~s}^{-1}\right.$, Klauk et al [16]). Also, measured mobilities depend on the thickness of the pentacene layer (higher for thinner films), as reported previously by Lee et al [17] and by us [14]. We have found that drain saturation currents are lowered with increasing pentacene film thickness, and a correction has to be applied to calculate the real OFET mobility $\mu_{\mathrm{OFET}}$ :

$$
\mu_{\mathrm{OFET}}=\mu_{\text {meas }} \frac{t_{\mathrm{p}}+t_{\mathrm{i}}}{t_{\mathrm{i}}}
$$

where $t_{\mathrm{p} / \mathrm{i}}$ are the thickness of the pentacene and gate insulator film, respectively. Equation (1) is equivalent to replacing the insulator thickness $t_{\mathrm{i}}$ with the total transistor thickness $t_{\mathrm{i}}+t_{\mathrm{p}}$ when calculating the gate capacitance $C_{\mathrm{i}}$. Since we use extremely thin gate insulators, $t_{\mathrm{i}} \approx 6.5 \mathrm{~nm}$, the factor $\left(t_{\mathrm{p}}+t_{\mathrm{i}}\right) / t_{\mathrm{i}}$ becomes very large: $\left(t_{\mathrm{p}}+t_{\mathrm{i}}\right) / t_{\mathrm{i}}=17.9$ for $t_{\mathrm{p}}=110 \mathrm{~nm}$. Therefore, measured mobilities here are smaller than those reported for pentacene on thicker gate insulators: Iino et al have used $t_{\mathrm{i}} \approx(85-190) \mathrm{nm}$ of $\mathrm{Ta}_{2} \mathrm{O}_{5}$, and Klauk et al have used $t_{\mathrm{i}} \approx 380 \mathrm{~nm}$ of an organic gate insulator. When we apply equation (1) to convert measured mobilities $\mu_{\text {meas }}$ into real OFET mobilities $\mu_{\mathrm{OFET}}$, we arrive at $\mu_{\mathrm{OFET}}=0.95$ (0.72) $\mathrm{cm}^{2} \mathrm{~V}^{-1} \mathrm{~s}^{-1}$ for $100(40) \mathrm{nm}$ pentacene/'citric acid', and $\mu_{\text {OFET }}=0.51 \mathrm{~cm}^{2} \mathrm{~V}^{-1} \mathrm{~s}^{-1}$ for the $110 \mathrm{~nm}$ pentacene/'water' insulator. Corrected mobilities vary by less than a factor of 2 , and are somewhat higher but similar to the mobilities reported by Iino et al [6] for pentacene on the more polar anodic $\mathrm{Ta}_{2} \mathrm{O}_{5}$.

\section{Conclusions}

We have shown that commercially available Mylar plastic sheets coated with a thin, sputtered Al film can be used for the manufacture of flexible organic transistors with both polymeric and low molecular weight organic semiconductors. Via anodization, extremely thin, high capacitance gate insulators consisting of $\mathrm{Al}_{2} \mathrm{O}_{3}$ can be prepared on the Mylar/Al films, using a simple procedure requiring no vacuum steps.

There is little difference between using very dilute citric acid, or pure water, as the anodization medium. However, water anodization is limited to very thin insulators, as otherwise, delamination of the Al film from the substrate occurs.

To realize the full potential of pentacene OFETs with extremely thin gate insulators, it is essential to deposit very thin pentacene films as well, because the total transistor thickness (insulator + semiconductor), not the insulator thickness alone, controls the saturation drain current. We speculate this may be a general phenomenon for transistors with non-Ohmic source/semiconductor contact. Further study is required to verify this.

\section{Acknowledgments}

The authors wish to thank the EPSRC for support under research grants GR/R88328/01 and GR/S02303/01.

\section{References}

[1] Sirringhaus H, Kawase T, Friend R H, Shimoda T, Inbasekaran M, Wu W and Woo E P 2000 Science 2902123

[2] Huitema H E A, Gelinck G H, van der Putten J B P H, Kuuijk K E, Hart K M, Cantatore E and de Leeuw D M 2002 Adv. Mater. 141201

[3] Street R A and Salleo A 2002 Appl. Phys. Lett. 812887

[4] Salleo A, Chabinyc M L, Yang M S and Street R A 2002 Appl. Phys. Lett. 814383

[5] Tate J, Rogers J A, Jones C D W, Vyas B, Murphy D W, Li W, Bao Z, Slusher R E, Dodabalapur A and Katz H E 2000 Langmuir 166054

[6] Iino Y, Inoue Y, Fujikake Y, Sato H, Kawakita M, Tokito S and Kikuchi H 2003 Japan. J. Appl. Phys. 42299

[7] Majewski L A, Grell M, Ogier S D and Veres J 2003 Organic Electronics 427

[8] Veres J, Ogier S D, Leeming S W, Cupertino D C and Khaffaf S M 2003 Adv. Funct. Mater. 13199

[9] Liang C W, Luo T C, Feng M S, Cheng H C and Su D 1996 Mater. Chem. Phys. $\mathbf{4 3} 166$

[10] Sharp D J, Panitz J K G, Merill R M and Haaland D M 1984 Thin Solid Films 111 227-34

[11] Lin Y P and Hwu J G 2001 Japan. J. Appl. Phys. 40 (part 1) 6788

[12] Abdou M S A, Orfino F P, Son Y and Holdcroft S 1997 J. Am. Chem. Soc. 1194518

[13] Koch N, Ghijsen J, Elschner A, Johnson R L, Pireaux J J, Schwartz J and Kahn A 2003 Appl. Phys. Lett. 8270

[14] Schroeder R, Majewski L A and Grell M 2003 Appl. Phys. Lett. 833210

[15] Bao Z, Dodabalapur A and Lovinger A J 1996 Appl. Phys. Lett. 694108

[16] Klauk H, Halik M, Zschieschang U, Schmid G, Radlik W and Weber W 2002 J. Appl. Phys. 925259

[17] Lee J, Kim K, Kim J H, Im S and Jung D-Y 2003 Appl. Phys. Lett. 824169 